Rapid Reviews COVID-19

\title{
Reviews of "Rapid 'mix and read' assay for scalable detection of SARS-CoV-2 antibodies in patient plasma"
}

Xi Chen ${ }^{1}$, Simin Xia ${ }^{2}$, Thomas Moran ${ }^{3}$, J. Andrew Duty ${ }^{4}$, Thomas Kraus ${ }^{4}$

${ }^{1}$ Harbin Institute of Technology, The HIT Center for Life Sciences, China, ${ }^{2}$ MVM, The HIT Center for Life Sciences, ${ }^{3}$ Icahn School of Medicine at Mount Sinai, Microbiology, USA, ${ }^{4}$ Icahn School of Medicine at Mount Sinai

Published on: Nov 15, 2020

DOI: $10.1162 / 2 \mathrm{e} 3983 f 5 . c 556 \mathrm{e} 628$

License: Creative Commons Attribution 4.0 International License (CC-BY 4.0). 
To read the original manuscript, click the link above.

Summary of Reviews: This pre-print offers a FRET-based diagnostic platform that is faster and less labor-intensive than ELISA-based diagnostics. The claims should be considered reliable.

Reviewer 1 (Xi Chen, Simin Xia) |

Reviewer 2 (Thomas Moran, J. Andrew Duty, Thomas Kraus)

$$
\begin{aligned}
& \text { RR:C19 Strength of Evidence Scale Key. }
\end{aligned}
$$

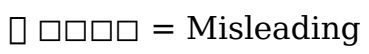

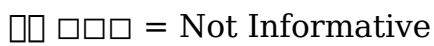

$$
\begin{aligned}
& \text { प्रा पि = Potentially Informative }
\end{aligned}
$$

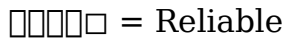

$$
\begin{aligned}
& \text { प्राप्र = Strong }
\end{aligned}
$$

To read the reviews, click the links below. 